\title{
Influence de la fréquence sur la production et l'acquisition des formes verbales du français : le cas des russophones adultes
}

\author{
Evgeniya Sergeeva \& Jean-Pierre Chevrot \\ Laboratoire LIDILEM - Université Stendhal Grenoble 3
}

\begin{abstract}
Le développement de la morphologie verbale d'une L2 est devenu un des sujets principaux de la recherche sur l'acquisition des langues étrangères. Il est possible de distinguer deux approches majeures à la question de l'acquisition de la morphologie verbale : une approche orientée à l'étude de l'acquisition des valeurs verbales et une seconde approche orientée à l'étude de l'acquisition des formes. Dans la première approche, le signe qu'une catégorie verbale est acquise est l'utilisation des marqueurs appropriés. Le problème qui se pose si l'on applique cette approche à la conjugaison orale française est que la majorité des changements catégoriels est exprimée par un changement de base dans une forme à flexion zéro.
\end{abstract}

Dans le cadre de l'approche orientée vers l'étude de l'interaction forme-valeur, les travaux se sont concentrés sur l'acquisition de l'accord verbal au présent à l'oral et à l'écrit. Il a été montré que les apprenants du FLE acquièrent plus rapidement le marquage par une flexion prononcée alors que la réalisation correcte des différences catégorielles exprimées par un changement de base (à l'exception des auxiliaires) est mise en place plus tardivement (Ågren, 2005 ; Bartning, 2004 ; Granget, 2005 ; Hedbor, 2005 ; Schlyter, 2005 ; Nouveau, 2007). Cependant, la variation des bases verbales en français ne se limite pas au marquage au présent. Aussi, la variation des bases verbales peut-elle devenir source d'erreurs dans la production des formes personnelles, temporelles et modales au cas où la flexion est bien sélectionnée. En effet, Debrenne (2006) a observé les erreurs sur la base dans un plus large éventail de formes verbales : à part l'accord au présent, les erreurs sur la base sont répertoriées pour le futur simple, le participe passé et le subjonctif.

D'autre part, il a été montré que la fréquence est un facteur influençant fortement l'acquisition et la production de la morphologie verbale en L1 et L2 (voir Ellis, 2002 pour une revue).

Malgré les avancées dans ces deux sujets de recherche, aucune étude, à notre connaissance, n'a cherché à observer l'acquisition d'un large éventail de bases verbales du français en relation avec les fréquences des formes et des bases verbales dans l'input des apprenants.

\section{Système de conjugaison du verbe français}

Nous présentons l'analyse qui a servi de fondement linguistique de notre recherche. Nous avons adopté ici la description de la conjugaison de Boyé (2000) qui se caractérise par une orientation vers l'oral et la mise en relation des bases verbales. Une des hypothèses principales de cette analyse est la maximisation de la base afin d'aboutir à l'identité des flexions orales pour tous les verbes. Ce choix suppose une plus grande importance de la supplétion des bases mais évite d'utiliser la notion du groupe de conjugaison.

Le paradigme d'un verbe est constitué, selon Boyé (2000), de 12 groupes de formes (Tab. 1). Dans la conjugaison d'un verbe donné, les formes d'un groupe utilisent toujours la même base. Par exemple, pour les formes du groupe «imparfait, présent $1 \& 2 \mathrm{pl}$. » le verbe «devoir » utilise une seule base : il devait, nous devons. Toutes les formes du groupe « subjonctif présent sg., 3pl. » utilisent aussi une seule base : qu'il doive, qu'ils doivent.

Un verbe donné peut utiliser une même base pour construire les formes de plusieurs groupes. Ainsi, «savoir » utilise une même base /sav/ pour toutes les formes du groupe «Imparfait, présent $1 \& 2 \mathrm{pl} /$ » : nous savons, ils savaient ainsi que pour le groupe «Présent 3pl. »: ils savent. La distinction de tous les 
deux groupes de formes est justifiée par le fait qu'il y a au moins un verbe qui utilise deux bases différentes pour ces deux groupes (Bonami \& Boyé, 2003).

En fonction des relations d'identité ou de différence de bases entre ces groupes, Bonami et Boyé (2003) distinguent 10 modèles d'alternances de bases verbales du français. Nous donnons ici pour l'exemple les modèles d'alternances des verbes « devoir » et « savoir» (Tab. 1) :

\begin{tabular}{|c|c|c|c|c|}
\hline \multirow{2}{*}{ Groupe de formes } & \multicolumn{2}{|c|}{ devoir } & \multicolumn{2}{c|}{ savoir } \\
\cline { 2 - 4 } & base & alternance & base & alternance \\
\hline imparfait, présent 1\&2pl. & $/ \mathrm{d} \partial /$ & $\mathrm{A}$ & $/ \mathrm{sav} /$ & $\mathrm{A}$ \\
\hline présent 3 pl. & $/ \mathrm{dwav} /$ & $\mathrm{B}$ & $/ \mathrm{sav} /$ & $\mathrm{A}$ \\
\hline présent sg. & $/ \mathrm{dwa} /$ & $\mathrm{C}$ & $/ \mathrm{s} /$ & $\mathrm{B}$ \\
\hline subjonctif présent 1\&2pl. & $/ \mathrm{d} \partial \mathrm{V} /$ & $\mathrm{A}$ & $/ \mathrm{saS} /$ & $\mathrm{C}$ \\
\hline subjonctif présent sg., 3pl. & $/ \mathrm{dwav} /$ & $\mathrm{B}$ & $/ \mathrm{saS} /$ & $\mathrm{C}$ \\
\hline impératif sg. & $/ \mathrm{dwa} /$ & $\mathrm{C}$ & $/ \mathrm{saS} /$ & $\mathrm{C}$ \\
\hline impératif pl. & $/ \mathrm{d} / \mathrm{v} /$ & $\mathrm{A}$ & $/ \mathrm{saS} /$ & $\mathrm{C}$ \\
\hline participe présent & $/ \mathrm{d} / \mathrm{v} /$ & $\mathrm{A}$ & $/ \mathrm{saS} /$ & $\mathrm{C}$ \\
\hline
\end{tabular}

Tab. 1. Exemple des modèles d'alternances de bases verbales

Cependant, dans l'établissement des modèles de conjugaison les auteurs laissent de côté 4 groupes de formes dont les bases ne se laissent pas déduire d'une autre base par une fonction unique. En effet, les relations entre les bases de ces quatre groupes de formes et les huit autres ne peuvent pas se réduire aux relations d'identité ou de supplétion de bases. Il s'agit plutôt de fonction phonologique de construction de base. Dans l'optique qui est la nôtre, nous avons jugé restrictif d'exclure de l'analyse les formes du futurconditionnel, du participe passé, de l'infinitif et du passé simple qui, sauf pour le dernier, sont fréquentes. D'autre part, nous ne savons rien pour l'instant des facultés des apprenants russophones à appliquer les règles de construction de bases. Pour cette raison, nous avons repris l'analyse de Bonami et Boyé pour obtenir 40 modèles d'alternances de bases, en analysant les bases des 4 groupes en question comme bases supplétives. Un travail systématique sur les fréquences listées dans la base Lexique 3 (New, 2001) aboutit au constat que ces modèles diffèrent tant par le nombre de bases utilisées que par la fréquence cumulée des modèles et des verbes représentant ces modèles.

Dans le cadre de cette extension du modèle linguistique de Boyé (2000), nous avons analysé la production des formes conjuguées du verbe français chez des apprenants russophones adultes recueillie à travers une tâche de production élicitée.

\section{Fréquence et alternance des bases en L2}

La question de l'influence de fréquence sur la production des formes verbales a souvent été examinée dans le cadre des modèles dits à double voie (Pinker \& Prince, 1988, 1991; Pinker, 1991, 1999; Marcus et al., 1992) qui postulent l'application de règles symboliques générant les formes régulières et d'un inventaire mémorisé de formes irrégulières. Dans ces modèles, les formes régulières et irrégulières différent par le rôle que joue la fréquence des formes dans leur acquisition et leur production : l'effet de fréquence devrait affecter seules les formes irrégulières. Cependant, les études de la production des formes verbales des langues à morphologie complexe ont démontré un effet de fréquence pour les formes irrégulières aussi bien que régulières (Meunier \& Marslen-Wilson, 2000; Matcovich, 1998; Ragnasdottir, Simonsen, 1997, 1998, 1999). Ces résultats ont été utilisés pour prouver qu'une dissociation tranchée entre les formes régulières et irrégulières ne peut pas être appliquée aux langues à morphologie verbale complexe, dont le français.

Nous voyons que les théories basées sur l'usage (Langacker, 1987; Bybee, 2001) ont une plus grande valeur explicative dans la description de l'acquisition des alternances de bases. Ces théories postulent que l'acquisition est basée sur les exemplaires, dont la mémorisation et la mise en relation aboutit à 
l'émergence de constructions dont le niveau d'abstraction est variable (Ellis, 2002). Par ailleurs, dans notre perspective les théories basées sur l'usage sont attractives par le fait qu'elles prédisent les relations entre les variantes des bases verbales. Selon Bybee $(1985,1991)$, certaines formes des paradigmes flexionnels peuvent être décrites comme « la base d'une autre forme + un marqueur ». Elle précise que ce genre de relations est possible également quand il s'agit d'alternances de base. Selon cette auteure, les enfants et les apprenants d'une L2 qui acquièrent la conjugaison commenceraient par utiliser une forme verbale qui sert à remplacer toutes les autres formes. Cette forme constituerait généralement la forme de base du paradigme $(\mathrm{FdB})$ dont peuvent être dérivées toutes les autres formes, car elle est fréquente et sémantiquement peu marquée. Ensuite les apprenants acquerraient les fonctions des autres formes et utiliseraient la forme de base pour les construire en ajoutant les marqueurs appropriés. Le plus souvent la FdB serait le singulier présent de l'indicatif.

\section{Tâche d'élicitation des formes verbales}

\subsection{Méthodologie et hypothèses}

Notre objectif était donc d'observer la production des formes conjuguées du verbe français avec un intérêt particulier au choix de bases verbales. Nous avons tenté de définir le rôle que jouent, dans la production juste et les erreurs, la fréquence des tokens et la présence dans le paradigme verbal de formes de base.

\subsubsection{Matériel verbal et tâche}

Dans ce but, nous avons conçu une tâche d'élicitation incluant des verbes présentant des modèles différents d'alternances de bases. Le classement de verbes par modèles d'alternances de bases et les fréquences nous ont permis de choisir trois verbes à haute fréquence cumulée et trois verbes à basse fréquence cumulée, tous issus de modèles d'alternances de bases différents (Tab. 2).

\begin{tabular}{|c|c|c|c|c|c|c|}
\hline \multirow{3}{*}{ Groupe de formes } & \multicolumn{6}{|c|}{ Fréquence cumulée } \\
\hline & \multicolumn{3}{|c|}{ Haute fréquence cumulée } & \multicolumn{3}{|c|}{ Basse fréquence cumulée } \\
\hline & faire & prendre & devoir & boire & craindre & traduire \\
\hline imparfait, présent 1\&2pl. & $\mathrm{A} / \mathrm{B}$ & A & A & A & A & A \\
\hline présent 3 pl. & $\mathrm{C}$ & $\mathrm{B}$ & $\mathrm{B}$ & $\mathrm{B}$ & $\mathrm{A}$ & $\mathrm{A}$ \\
\hline présent sg. & $\mathrm{D}$ & $\mathrm{C}$ & $\mathrm{C}$ & $\mathrm{C}$ & $\mathrm{B}$ & $\mathrm{B}$ \\
\hline subjonctif présent $1 \& 2 \mathrm{pl}$. & $\mathrm{E}$ & A & $\mathrm{A}$ & $\mathrm{A}$ & $\mathrm{A}$ & $\mathrm{A}$ \\
\hline subjonctif présent sg., $3 \mathrm{pl}$. & $\mathrm{E}$ & $\mathrm{B}$ & $\mathrm{B}$ & $\mathrm{B}$ & $\mathrm{A}$ & $\mathrm{A}$ \\
\hline impératif sg. & $\mathrm{D}$ & $\mathrm{C}$ & $\mathrm{C}$ & $\mathrm{C}$ & $\mathrm{B}$ & $\mathrm{B}$ \\
\hline impératif pl. & $\mathrm{A}$ & $\mathrm{A}$ & $\mathrm{A}$ & $\mathrm{A}$ & A & A \\
\hline participe présent & $\mathrm{A}$ & $\mathrm{A}$ & $\mathrm{A}$ & $\mathrm{A}$ & $\mathrm{A}$ & $\mathrm{A}$ \\
\hline futur-conditionnel & $\mathrm{F}$ & $\mathrm{D}$ & $\mathrm{A}$ & $\mathrm{C}$ & $\mathrm{C}$ & $\mathrm{B}$ \\
\hline passé simple & $\mathrm{G}$ & $\mathrm{E}$ & $\mathrm{D}$ & $\mathrm{D}$ & $\mathrm{D}$ & $\mathrm{C}$ \\
\hline infinitif & $\mathrm{D}$ & $\mathrm{D}$ & $\mathrm{E}$ & $\mathrm{C}$ & $\mathrm{C}$ & B \\
\hline participe passé & $\mathrm{D}$ & $\mathrm{E}$ & $\mathrm{D}$ & $\mathrm{D}$ & $\mathrm{B}$ & $\mathrm{B}$ \\
\hline
\end{tabular}

Tab. 2. Vrais verbes élicités

Les formes temporelles et modales à éliciter ont été choisies en fonction des connaissances des apprenants. L'analyse des manuels utilisés pendant les cours des apprenants concernés nous a permis de voir quels temps et quels modes ont été appris et lesquels ne l'ont pas été. Les formes à éliciter étaient : la $3^{\text {ème }}$, la $4^{\text {ème }}$ et la $6^{\text {ème }}$ personnes du présent de l'indicatif, la $3^{\text {ème }}$ personne du subjonctif présent, la $3^{\text {ème }}$ personne du futur simple, le participe passé et l'infinitif. Ces temps-personnes ont été choisis pour leur capacité à représenter l'éventail des variantes des bases verbales. 


\subsubsection{Sujets}

Cette tâche d'élicitation a été proposée à un groupe de 30 apprenants de niveaux différents en français. Le niveau des sujets a été établi à partir d'un questionnaire d'input. Ce questionnaire est composé de 17 questions rédigées en russe, il est une adaptation du travail de Payre-Ficout (2007) conçu pour des apprenants d'anglais langue étrangère. Les questions traitent de la présence des langues étrangères dans l'environnement langagier, passé ou présent, scolaire ou familial, des sujets. En nous basant sur ces questionnaires, nous avons sélectionné 30 sujets répartis sur deux niveaux différents : deuxième année spécialité journalisme et histoire $(\mathrm{N}=15)$ versus quatrième année spécialité lettres $(\mathrm{N}=15)$.

\subsubsection{Procédure}

Chaque sujet a passé la tâche de production individuellement. La production de chaque forme cible a été élicitée deux fois. Les verbes ont été présentés dans un ordre aléatoire ainsi que les formes de tempspersonne ciblées. La consigne a été présentée oralement et les réponses ont été enregistrées.

\subsubsection{Hypothèses}

La tâche de production a été conçue pour vérifier les prédictions suivantes en ce qui concerne l'influence de la fréquence :

- L'acquisition du marquage par une flexion prononcée devrait être plus facile pour les apprenants russophones.

- Un des principaux facteurs agissant sur la sélection des bases verbales est leur fréquence de surface ainsi que la fréquence de surface d'autres bases compétitives du même verbe.

- La production des formes verbales est influencée par leur fréquence de surface de sorte que le taux de correction des formes plus fréquentes sera supérieur à celui des formes rares.

- Les apprenants avancés, qui ont eu une plus grande exposition à la langue française, devraient être plus sensibles à l'effet de fréquence verbale et produire plus correctement les formes fléchies fréquentes ainsi que de sélectionner les bases fréquentes plus facilement.

- Dans les formes erronées construites avec une base et une forme existantes, une forme ou une base plus fréquente devrait remplacer une forme ou une base moins fréquente.

- Le verbe français possède plusieurs FdB dans l'acception de Bybee (1985). Le statut canonique de plusieurs formes peut également être un facteur affectant la sélection de base verbales.

- Le verbe français possède plusieurs FdB dans l'acception de Bybee. La production juste de ces formes ne serait pas sensible à la fréquence et ces formes devraient être utilisées pour en remplacer d'autres. Pour les apprenants du FLE, ce serait les formes du singulier présent et de l'infinitif (cf. Thomas, 2005).

\subsection{Décompte des fréquences : cas du français langue étrangère}

A notre connaissance, peu d'études ont essayé de comparer les fréquences d'input réel des apprenants d'une L2 et l'output produit par ces mêmes apprenants. Cependant, comme le notent Gor et Chernigovskaya (2003), les apprenants d'une L2 en milieu académique ont un accès limité aux fréquences verbales, par comparaison aux enfants apprenant leur langue maternelle. Ces deux auteures proposent un calcul de fréquences basé sur la méthode de langue utilisée dans le groupe d'apprenants américains du russe qu'elles observent. Pour ce qui est des études de l'acquisition du FLE, Thomas (2005) a réalisé le décompte des fréquences à partir des énoncés de l'enseignante dans un corpus d'entretiens guidés. 
A la différence des apprenants du russe dont les productions ont été analysées par Gor et Chernigovskaya (2003), les apprenants de notre groupe expérimental n'étaient pas des débutants. En outre, le questionnaire d'input a montré qu'ils ont d'autres sources d'input que les cours (pour les apprenants du niveau élevé en français). De ce fait, l'établissement des fréquences à partir d'un corpus basé sur le manuel de français utilisé en classe ne représente qu'une partie de l'input. Nous allons cependant utiliser les fréquences verbales de ce corpus, tout en les comparant aux fréquences répertoriées dans la base de données Lexique 3 (New, 2001).

Dans notre cas, tous les apprenants ont suivi les cours de français basés sur la méthode «Le Nouveau sans frontières » $(N S F)$. Le groupe d'apprenants de niveau bas $(N 1)$ a achevé deux niveaux de la méthode, le groupe d'apprenants de niveau élevé (N2) en a achevé quatre. Pour constituer le corpus, les manuels et les cahiers d'exercices ont été entièrement scannés et nous avons mis en œuvre un procédé de reconnaissance optique des caractères pour constituer un corpus numérique. Nous avons inclus les pages en entier sauf les numéros de pages et les titres. Le décompte des fréquences a concerné uniquement les formes écrites dans les manuels. Nous avons sciemment laissé de côté les formes qui étaient à produire dans les exercices. Nous avons ensuite appliqué le test de Pearson pour calculer les corrélations entre les fréquences répertoriées dans Lexique 3 et les fréquences obtenues à partir des corpus. Les indices de corrélation sont élevés (Tab. 3).

\begin{tabular}{|c|c|}
\hline $\begin{array}{c}\text { Unité de quantification de } \\
\text { fréquence }\end{array}$ & $\begin{array}{c}\text { Indice de corrélation } \\
\boldsymbol{N S F}-\text { Lexique 3 }\end{array}$ \\
\hline forme fléchie cible & $0,892(\mathrm{p}<0,001)$ \\
\hline $\begin{array}{c}\text { homophones de la forme cible dans } \\
\text { le paradigme du verbe conjugué }\end{array}$ & $0,990(\mathrm{p}<0,001)$ \\
\hline base & $0,989(\mathrm{p}<0,001)$ \\
\hline
\end{tabular}

Tab. 3. Corrélation des fréquences obtenues de l'analyse du corpus NSF et des fréquences répertoriées dans Lexique 3 (New, 2001)

Ce résultat signifie que, globalement, la fréquence générale des formes verbales est très bien représentée dans leurs occurrences dans les manuels.

\subsection{Résultats}

Nous présentons ici une analyse des résultats de la tâche de production en les mettant en relation avec les prédictions que nous avons émises par rapport au rôle de la fréquence dans les productions des formes verbales du FLE.

Le Tableau suivant présente les taux moyens de production de différentes combinaisons base - flexion en fonction de leur justesse (Tab. 4).

\begin{tabular}{|l|c|c|}
\hline Combinaison base - flexion & Exemple & Taux moyen \\
\hline forme fléchie juste & /il bwara/ & $57.5 \%$ \\
\hline base incorrecte + flexion correcte & /il byvra/ & $28.8 \%$ \\
\hline base correcte + flexion incorrecte & /il bwarE/ & $3.8 \%$ \\
\hline tout incorrect & /il byvrE/ & $9.9 \%$ \\
\hline Total & & $\mathbf{1 0 0 \%}$ \\
\hline
\end{tabular}

Tab. 4. Combinaisons base - flexion en fonction de leur justesse

Nous voyons que la majorité d'erreurs relève du choix de la base, alors que les flexions sont bien sélectionnées. Ce résultat est en accord avec les travaux antérieurs où il a été démontré que, pour les apprenants du FLE, le marquage par un changement de base verbale est plus long à mettre en place que le marquage par une flexion prononcée (Ågren, 2005 ; Bartning, 2004 ; Granget, 2005 ; Hedbor, 2005 ; Schlyter, 2005). 
Nous avons calculé les taux moyens de production juste des formes fléchies et de sélection des bases justes selon la formule suivante :

Nombre de forme fléchies / de bases correctes

Nombre total de formes élicitées - Nombre de non-réponses

$\mathrm{x} 100 \%$

Comme le fait apparaître cette formule, nous n'avons pas pénalisé les non-réponses du fait qu'elles ne représentent que $0.6 \%$ du total de formes élicitées dans la tâche.

\subsubsection{Effet de la fréquence des tokens}

- Effet de la fréquence des tokens sur les productions justes

Pour voir s'il y a un effet global de fréquence, nous avons comparé les taux moyens de production de formes fléchies justes et de sélection de bases justes des verbes à haute et basse fréquence cumulée. Les taux moyens et les écarts-types sont listés dans le Tableau 5.

\begin{tabular}{|c|c|c|c|c|c|}
\hline \multicolumn{2}{|c|}{ Production de formes fléchies justes } & \multicolumn{3}{c|}{ Sélection de bases justes } \\
\hline $\begin{array}{c}\text { verbes } \\
\text { fréquents }\end{array}$ & $\begin{array}{c}\text { verbes } \\
\text { rares }\end{array}$ & $\begin{array}{c}\text { Significativité du } \\
\text { t-test }\end{array}$ & $\begin{array}{c}\text { verbes } \\
\text { fréquents }\end{array}$ & $\begin{array}{c}\text { verbes } \\
\text { rares }\end{array}$ & $\begin{array}{c}\text { Significativité } \\
\text { du t-test }\end{array}$ \\
\hline 57.88 & 45.08 & $\mathrm{t}=2.2609$ & 88.22 & 64.22 & $\mathrm{t}=4.0267$ \\
$(27.39)$ & $(25.39)$ & $\mathrm{p}=0.0275$ & $(21.19)$ & $(15.28)$ & $\mathrm{p}<0.001$ \\
\hline
\end{tabular}

Tab. 5. Taux de production des formes fléchies justes et des bases justes des verbes à haute et à basse fréquence cumulée (moyennes et écarts-types)

Nous voyons sur ce tableau que les taux moyens de correction sont plus élevés dans le cas des verbes à haute fréquence cumulée. Ces résultats sont significatifs tant pour la production des formes fléchies que pour la sélection des bases.

- Volume de l'input et effet de la fréquence des tokens sur les productions justes

Si nous observons l'effet global de la fréquence dans les productions de chaque niveau des apprenants (N1 versus N2), nous voyons que l'effet de fréquence persiste uniquement dans les productions du groupe N2 (Tab. 6). Comme dans l'analyse globale, ces résultats sont significatifs pour la production des formes fléchies justes ainsi que pour la sélection des bases justes.

\begin{tabular}{|c|c|c|c|c|c|c|}
\hline \multirow{2}{*}{ Niveau } & \multicolumn{2}{|c|}{ Production de formes fléchies } & \multicolumn{3}{c|}{$\begin{array}{c}\text { Sélection } \\
\text { justes }\end{array}$} \\
\cline { 2 - 7 } & $\begin{array}{c}\text { verbes justes } \\
\text { fréquents }\end{array}$ & $\begin{array}{c}\text { verbes } \\
\text { rares }\end{array}$ & $\begin{array}{c}\text { Significativité } \\
\text { du t-test }\end{array}$ & $\begin{array}{c}\text { verbes } \\
\text { fréquents }\end{array}$ & $\begin{array}{c}\text { verbes } \\
\text { rares }\end{array}$ & $\begin{array}{c}\text { Significativité } \\
\text { du t-test }\end{array}$ \\
\hline \multirow{2}{*}{$\mathrm{N} 1$} & 42.26 & 41.85 & $\mathrm{t}=0.982$ & 50.15 & 47.59 & $\mathrm{t}=0.845$ \\
& $(16.04)$ & $(12.68)$ & $\mathrm{p}=0.33$ & $(15.69)$ & $(10.42)$ & $\mathrm{p}=0.41$ \\
\hline \multirow{2}{*}{$\mathrm{N} 2$} & 77.26 & 63.90 & $\mathrm{t}=4.056$ & 82.22 & 67.37 & $\mathrm{t}=2.764$ \\
& $(13.37)$ & $(16.26)$ & $\mathrm{p}<0.001$ & $(11.53)$ & $(12.84)$ & $\mathrm{p}=0.009$ \\
\hline
\end{tabular}

Tab. 6. Taux de production des formes fléchies justes et de sélection de bases justes des verbes à haute et basse fréquence cumulée en fonction du niveau des apprenants (moyennes et écarts-types)

Cette tendance se confirme par une analyse des corrélations de Pearson entre les taux de production des formes fléchies justes et de sélection des bases justes et les fréquences des ces unités en fonction du niveau des apprenants (Tab. 7). 


\begin{tabular}{|c|c|c|c|c|}
\hline \multirow{2}{*}{ Niveau } & \multicolumn{2}{|c|}{$\begin{array}{c}\text { Production de formes fléchies } \\
\text { justes }\end{array}$} & \multicolumn{2}{c|}{$\begin{array}{c}\text { Sélection } \\
\text { de bases justes }\end{array}$} \\
\cline { 2 - 5 } & $\begin{array}{c}\text { Fréquence } \\
\text { forme cible }\end{array}$ & $\begin{array}{c}\text { Fréquence } \\
\text { base }\end{array}$ & $\begin{array}{c}\text { Fréquence } \\
\text { forme cible }\end{array}$ & $\begin{array}{c}\text { Fréquence } \\
\text { base }\end{array}$ \\
\hline \multirow{2}{*}{$\mathrm{N} 1$} & $\begin{array}{c}0.465^{*} \\
(\mathrm{p}<0.001)\end{array}$ & $\begin{array}{c}0.448^{*} \\
(\mathrm{p}<0.001)\end{array}$ & $\begin{array}{c}0.237^{*} \\
(\mathrm{p}=0.045)\end{array}$ & $\begin{array}{c}0.344^{*} \\
(\mathrm{p}=0.003)\end{array}$ \\
\hline $\mathrm{N} 2$ & $\begin{array}{c}0.619^{*} \\
(\mathrm{p}<0.001)\end{array}$ & $\begin{array}{c}0.593^{*} \\
(\mathrm{p}<0.001)\end{array}$ & $\begin{array}{c}0.048 \\
(\mathrm{p}=0.690)\end{array}$ & $\begin{array}{c}0.041 \\
(\mathrm{p}=0.730)\end{array}$ \\
\hline
\end{tabular}

Tab. 7. Corrélation des taux de production correcte et des fréquences $N S F$ en fonction du niveau des apprenants. (* - corrélation significative à $\mathrm{p} \leq 0.05$ )

Pour les apprenants du groupe N1, les corrélations entre les taux de production des formes correctes et la fréquence de surface des formes elles-mêmes ainsi qu'avec la fréquence des bases sont significatives. Nous observons également des corrélations significatives entre les taux de sélection de bases correctes et les fréquences de ces bases, ainsi qu'avec la fréquence des formes fléchies correspondantes.

Dans les productions du groupe N2, nous notons des corrélations positives et fortes entre les taux de correction des formes fléchies et leurs fréquences de surface, ainsi que les fréquences des bases correspondantes. Cependant, les corrélations entre les taux de sélection des bases justes et les fréquences des formes correspondantes ainsi que des bases elles-mêmes ne sont pas significatives.

Pour vérifier si l'effet de fréquence est évolutif, nous avons comparé les indices de corrélation qui sont significatifs dans les deux groupes des apprenants. Le résultat obtenu montre que, dans les productions du groupe N2, les corrélations sont plus fortes s'agissant de l'interaction de la production des formes fléchies justes avec la fréquence des formes cibles $(\mathrm{p}=0.033)$ ainsi qu'avec la fréquence des bases cibles $(p=0.047)$. Globalement, les formes et les bases fréquentes ont tendance à être produites correctement et cet effet de fréquence augmente avec le niveau des apprenants en FLE.

- Effet de la fréquence des tokens et les patterns d'erreurs

Pour vérifier si la fréquence des tokens peut expliquer les erreurs de substitution des bases, nous avons analysé les erreurs construites avec les bases existantes. Ces erreurs représentent 68.02\% (619/910) de toutes les erreurs. Les formes obtenues peuvent être tant des formes existantes (il /dwav/ remplacé par il /dwa/) que des formes inexistantes (il /dwav/ remplacé par il /dəvwa/). Sur ces erreurs, 262 erreurs $(42.3 \%)$ sont à structure complexe (base + flexion prononcée) et parmi ces dernières, $98(34.4 \%)$ sont des formes inexistantes. Les 357 erreurs restantes (57.8\%) sont à structure simple (base + flexion zéro). Dans ces erreurs, le taux des formes inexistantes est beaucoup plus bas : 17/357 (4.8\%).

Dans le Tableau 8 apparaissent les patterns d'erreurs en fonction de la structure des formes cibles et des formes substituts : forme simple (FS) versus forme complexe (FC), leur fréquence d'apparition et le pourcentage d'erreurs représenté.

\begin{tabular}{|c|c|c|c|}
\hline Type d'erreur & Nombre & $\mathbf{\%}$ & Exemple \\
\hline$F S$ pour $F S$ & 290 & 46.84 & prã pour prEn \\
\hline$F S$ pour $F C$ & 67 & 10.82 & $d w a$ pour dəvõ \\
\hline$F C$ pour $F C$ & 162 & 26.17 & fErE pour fora \\
\hline$F C$ pour $F S$ & 100 & 16.16 & dave pour $d y$ \\
\hline Total & $\mathbf{6 1 9}$ & $\mathbf{1 0 0 \%}$ & \\
\hline
\end{tabular}

Tab. 8. Formes erronées construites à partir des bases et des flexions existantes

Les fréquences moyennes et les écarts-types des formes et des bases cibles et substituts sont résumés dans le Tableau 9. Les fréquences sont calculées à partir du corpus NSF correspondant à l'input du groupe d'apprenants. Toutes les fréquences sont données sur 100.000 mots. 


\begin{tabular}{|c|c|c|c|c|}
\hline $\begin{array}{c}\text { Pattern } \\
\text { d'erreur }\end{array}$ & $\begin{array}{c}\text { fréquence } \\
\text { forme cible }\end{array}$ & $\begin{array}{c}\text { fréquence forme } \\
\text { substitut }\end{array}$ & $\begin{array}{c}\text { fréquence base } \\
\text { cible }\end{array}$ & $\begin{array}{c}\text { fréquence base } \\
\text { substitut }\end{array}$ \\
\hline$F S$ pour $F S$ & $8.83(18.38)$ & $38.91(58.08)$ & $16.28(45.79)$ & $80.72(142.44)$ \\
\hline$F S$ pour $F C$ & $8.17(30.84)$ & $53.05(71.76)$ & $35.61(56.48)$ & $133.05(199.38)$ \\
\hline$F C$ pour $F C$ & $9.26(28.05)$ & $5.36(7.71)$ & $36.79(50.91)$ & $84.18(157.67)$ \\
\hline$F C$ pour $F S$ & $10.75(13.95)$ & $14.41(29.40)$ & $30.53(89.22)$ & $46.01(101.55)$ \\
\hline
\end{tabular}

Tab. 9. Fréquences des formes et des bases cibles et substituts des quatre patterns d'erreurs (moyennes et écarts-types)

Une comparaison des fréquences des unités cibles et substituts fait apparaître que les formes substituts à structure simple sont plus fréquentes que les cibles simples qu'elles remplacent (pattern FS pour FS). Pour ce pattern d'erreurs, la différence est significative entre les fréquences des formes $(\mathrm{t}=7.317$, $\mathrm{p}<0.001)$ ainsi qu'entre les fréquences des bases $(\mathrm{t}=8.376, \mathrm{p}<0.001)$. Lorsqu'une forme simple remplace une forme complexe (pattern FS pour FC), le substitut est plus fréquent que la cible tant par la fréquence de la forme fléchie $(\mathrm{t}=4.668, \mathrm{p}<0.001)$ que par la fréquence de la base $(\mathrm{t}=3.820, \mathrm{p}=0.031)$. Dans le cas du pattern $F C$ pour $F C$, nous n'avons pas observé de différence significative entre les fréquences des formes fléchies substituts et cibles à structure complexe $(\mathrm{t}=1.663, \mathrm{p}=0.098)$. Cependant, les bases des substituts à structure complexe se sont révélées plus fréquentes que les bases des cibles à structure complexe $(\mathrm{t}=4.014, \mathrm{p}<0.001)$. Lorsqu'une forme à structure complexe remplace une forme à structure simple (pattern $F C$ pour $F S$ ), la différence n'est significative ni entre les fréquences des formes fléchies $(\mathrm{t}=0.663$, $\mathrm{p}=0.508)$, ni entre les fréquences des bases $(\mathrm{t}=1.432, \mathrm{p}=0.154)$.

Globalement, les formes et les bases plus fréquentes ont tendance à remplacer les formes et les bases moins fréquentes. Cet effet de fréquence est plus prononcé lorsque le substitut est une forme à structure simple.

\subsubsection{Quelles formes de base du verbe pour les apprenants du FLE ?}

Afin de voir quelles formes sont utilisées comme formes de base de la conjugaison par les apprenants russophones, nous avons analysé les formes de temps-personne élicitées dans la tâche suivant les indices d'une FdB listés ci-dessous. Selon Bybee (1985), les FdB du paradigme verbal sont définies comme les formes qui sont sémantiquement peu marquées. D'une part, elles doivent être fréquentes et acquises tôt. En effet, une fréquence élevée suppose une grande résistance aux erreurs. D'autre part, les formes de base doivent être utilisées pour construire d'autres formes du paradigme verbal. Pour ce dernier indice, nous voyons deux variantes possibles : la forme de base peut remplacer directement une forme cible fléchie ou elle peut être utilisée comme base à laquelle s'ajoute le marqueur approprié pour la cible.

\section{- Quelles formes résistent le plus aux erreurs?}

Pour identifier les FdB les plus plausibles parmi les 7 formes élicitées dans la tâche, nous avons commencé par analyser les taux de correction des formes et des bases pour chaque forme cible. Les résultats sont résumés dans le Tableau 10 en fonction des formes cibles et du niveaux des apprenants (N1 versus N2).

Ce tableau montre que trois formes - le 3PR, l'INF et le PP (Formes1) - se distinguent des autres (Formes2) par un taux de productions justes plus élevé. Nous avons vérifié, d'une part, s'il y avait des différences entre les taux de correction dans le groupe Formes1, et, d'autre part, si les différences avec les taux de correction du groupe Formes2 étaient significatives.

Dans les productions du N1, les taux de correction des formes fléchies ne sont pas significativement différents entre les 3PR et l'INF ( $\mathrm{t}=1.086, \mathrm{p}=0.287)$, le 3PR et le PP $(\mathrm{t}=1.378, \mathrm{p}=0.178)$, le PP et l'INF $(\mathrm{t}=0.123, \mathrm{p}=0.903)$. Les taux de correction des temps-personnes du groupe Formes1 (3PR, INF, PP) sont systématiquement supérieurs aux taux de correction des temps-personnes du groupe Formes2 (4PR, 6PR, 3SUBJ, 3FUT) $(\mathrm{p}<0,01)$. 


\begin{tabular}{|c|c|c|c|c|}
\hline \multirow{2}{*}{ Forme cible } & \multicolumn{2}{|c|}{ Production des formes fléchies justes } & \multicolumn{2}{c|}{ Sélection des bases justes } \\
\cline { 2 - 5 } & N1 & N2 & N1 & N2 \\
\hline 3PR & $74.44(16.51)$ & $92.20(8.59)$ & $75.45(16.51)$ & $92.23(8.60)$ \\
\hline INF & $65.55(16.87)$ & $95.54(16.92)$ & $65.57(27.08)$ & $95.56(7.62)$ \\
\hline PP & $64.42(23.71)$ & $84.98(14.84)$ & $64.46(22.82)$ & $85.01(15.17)$ \\
\hline 4PR & $44.44(21.10)$ & $70.54(25.29)$ & $45.56(17.78)$ & $70.01(16.91)$ \\
\hline 3FUT & $23.89(17.51)$ & $62.77(25.17)$ & $43.89(21.25)$ & $70.57(17.50)$ \\
\hline 6PR & $22.22(22.82)$ & $65.00(15.17)$ & $27.22(22.60)$ & $66.11(14.94)$ \\
\hline 3SUBJ & $21.12(27.08)$ & $52.22(7.62)$ & $24.99(19.92)$ & $52.78(24.13)$ \\
\hline
\end{tabular}

Tab. 10. Taux de production des formes justes et des bases justes en fonction des formes cibles et des niveaux (moyennes et écarts-types)

$3 P R-3^{\text {ème }}$ personne du présent, $I N F-$ infinitif, $P P-$ participe passé, $4 P R-4^{\text {ème }}$ personne du présent, $3 F U T-3^{\text {ème }}$ personne du futur simple, $6 P R-6^{\text {ème }}$ personne du présent, $3 S U B J-3^{\text {ème }}$ personne du subjonctif.

Le pattern observé dans les productions du groupe N2 est pratiquement le même. La différence n'est pas significative entre les taux de production de formes fléchies justes du 3PR et de l'INF ( $\mathrm{t}=-1.123$, $\mathrm{p}=0.271)$, du 3PR et du PP $(\mathrm{t}=1.603, \mathrm{p}=0.120)$. La différence est significative entre le PP et l'INF ( $\mathrm{t}=-$ $2.408, \mathrm{p}=0.022$ ). Comme dans les productions du groupe $\mathrm{N} 1$, nous retrouvons une différence significative systématique entre les taux de correction des temps-personnes du groupe Formes1 et du groupe Formes2 $(\mathrm{p}<0,02)$.

Ces résultats montrent que les formes en question - le 3PR, l'INF et le PP - se distinguent par un taux de production juste élevé chez les apprenants des deux niveaux. Ce qui suppose que les formes en question résistent aux erreurs significativement mieux que les autres formes élicitées, même dans les productions du groupe N1.

Pour vérifier si un haut taux de correction de ces formes est basé sur leur fréquence, nous avons analysé les corrélations entre les taux de correction des formes et des bases cibles et leurs fréquences dans chaque groupe de sujets. Le résumé des coefficients de corrélation de Pearson est donné dans le tableau suivant (Tab. 11).

\begin{tabular}{|c|c|c|c|c|c|c|c|c|}
\hline & Niveau & $3 P R$ & $P P$ & $I N F$ & $4 P R$ & $6 P R$ & $3 S U B J$ & $3 F U T$ \\
\hline \multirow{2}{*}{$\begin{array}{l}\text { Taux formes } \\
\text { correctes } \mathrm{x} \\
\text { Fréquence } \\
\text { forme }\end{array}$} & N1 & $\begin{array}{c}-0.197 \\
p=0.708\end{array}$ & $\begin{array}{c}0.517 \\
p=0.085\end{array}$ & $\begin{array}{c}0.391 \\
p=0.444\end{array}$ & $\begin{array}{c}0.512 \\
p=0.089\end{array}$ & $\begin{array}{c}0.139 \\
p=0.668\end{array}$ & $\begin{array}{c}0.769^{*} \\
p=0.003\end{array}$ & $\begin{array}{l}-0.789^{*} \\
p=0.002\end{array}$ \\
\hline & N2 & $\begin{array}{c}0.488 \\
p=0.326\end{array}$ & $\begin{array}{c}0.513 \\
p=0.088\end{array}$ & $\begin{array}{c}0.642 \\
p=0.170\end{array}$ & $\begin{array}{c}0.446 \\
p=0.146\end{array}$ & $\begin{array}{c}0.756^{*} \\
p=0.004\end{array}$ & $\begin{array}{c}0.676^{*} \\
\mathrm{p}=0.016\end{array}$ & $\begin{array}{c}0.300 \\
p=0.343\end{array}$ \\
\hline \multirow{2}{*}{$\begin{array}{l}\text { Taux formes } \\
\text { correctes } \mathrm{x} \\
\text { Fréquence } \\
\text { base }\end{array}$} & N1 & $\begin{array}{c}-0.001 \\
p=0.998\end{array}$ & $\begin{array}{c}0.433 \\
p=0.160\end{array}$ & $\begin{array}{c}0.549 \\
p=0.259\end{array}$ & $\begin{array}{c}0.418 \\
p=0.176\end{array}$ & $\begin{array}{c}0.110 \\
p=0.733\end{array}$ & $\begin{array}{c}0.554 \\
p=0.062\end{array}$ & $\begin{array}{c}-0.184 \\
p=0.567\end{array}$ \\
\hline & N2 & $\begin{array}{c}0.588 \\
p=0.220\end{array}$ & $\begin{array}{c}0.549 \\
p=0.065\end{array}$ & $\begin{array}{c}0.717 \\
p=0.109\end{array}$ & $\begin{array}{c}0.597^{*} \\
\mathrm{p}=0.040\end{array}$ & $\begin{array}{c}0.670^{*} \\
p=0.017\end{array}$ & $\begin{array}{c}0.563 \\
p=0.057\end{array}$ & $\begin{array}{c}-0.015 \\
p=0.946\end{array}$ \\
\hline \multirow{2}{*}{$\begin{array}{l}\text { Taux bases } \\
\text { correctes } \mathrm{x} \\
\text { Fréquence } \\
\quad \text { forme }\end{array}$} & N1 & $\begin{array}{c}-0.197 \\
p=0.708\end{array}$ & $\begin{array}{c}0.517 \\
\mathrm{p}=0.085\end{array}$ & $\begin{array}{c}0.433 \\
p=0.160\end{array}$ & $\begin{array}{c}0.538 \\
p=0.071\end{array}$ & $\begin{array}{c}0.113 \\
\mathrm{p}=0.727\end{array}$ & $\begin{array}{c}0.704^{*} \\
\mathrm{p}=0.011\end{array}$ & $\begin{array}{l}-0.660 * \\
p=0.019\end{array}$ \\
\hline & N2 & $\begin{array}{c}0.488 \\
p=0.326\end{array}$ & $\begin{array}{c}0.513 \\
p=0.088\end{array}$ & $\begin{array}{c}0.549 \\
p=0.065\end{array}$ & $\begin{array}{c}0.416 \\
p=0.179\end{array}$ & $\begin{array}{c}0.716^{*} \\
p=0.009\end{array}$ & $\begin{array}{c}0.655^{*} \\
p=0.021\end{array}$ & $\begin{array}{c}0.494 \\
p=0.102\end{array}$ \\
\hline \multirow{2}{*}{$\begin{array}{c}\text { Taux bases } \\
\text { correctes x } \\
\text { Fréquence } \\
\text { base }\end{array}$} & N1 & $\begin{array}{c}-0.001 \\
p=0.998\end{array}$ & $\begin{array}{c}0.391 \\
p=0.444\end{array}$ & $\begin{array}{c}0.549 \\
p=0.259\end{array}$ & $\begin{array}{c}0.436 \\
p=0.157\end{array}$ & $\begin{array}{c}0.099 \\
p=0.760\end{array}$ & $\begin{array}{c}0.637^{*} \\
\mathrm{p}=0.026\end{array}$ & $\begin{array}{c}0.233 \\
p=0.466\end{array}$ \\
\hline & N2 & $\begin{array}{c}0.588 \\
p=0.220\end{array}$ & $\begin{array}{c}0.582 \\
p=0.225\end{array}$ & $\begin{array}{c}0.652 \\
p=0.185\end{array}$ & $\begin{array}{c}0.570 * \\
p=0.053\end{array}$ & $\begin{array}{c}0.640^{*} \\
p=0.025\end{array}$ & $\begin{array}{c}0.542 \\
p=0.069\end{array}$ & $\begin{array}{c}0.068 \\
p=0.835\end{array}$ \\
\hline
\end{tabular}

Tab. 11. Corrélations entre les taux de production des formes fléchies justes et des bases justes avec les fréquences des tokens $(*$ - corrélation est significative à $\mathrm{p} \leq 0.05)$. 
Nous observons des corrélations significatives fortes et positives pour trois formes du groupe Formes2 (4PR, 6PR et 3SUBJ). Il est intéressant de rendre compte que la corrélation fréquence - performance est négative pour la forme du 3FUT dans les productions du groupe N1. Il est à souligner que, dans l'analyse des résultats des tests de corrélation pour les trois formes candidates au statut de FdB (3PR, INF, PP), les corrélations fréquence - taux de correction ne sont pas significatives.

Ainsi, comme nous le prévoyions pour une $\mathrm{FdB}$, le taux des productions justes de ces formes est élevé sans corrélation à la fréquence des formes et des bases cibles. Cependant, bien que le PP soit globalement bien réussi sans corrélation avec sa fréquence, nous estimons qu'il ne remplit pas le premier critère avancé par Bybee. Le PP est, en effet, sémantiquement marqué. Nous ne retenons donc que deux formes le 3PR et l'INF - pour l'analyse ultérieure.

\section{- Le 3PR et l'INF sont-ils utilisés pour construire le paradigme verbal ?}

Le dernier critère proposé par Bybee (1985) pour déterminer une forme de base est le fait qu'elle devrait être utilisée pour construire le paradigme verbal. La majeure source de preuve de l'utilisation des formes pour la construction du paradigme relève de l'analyse des erreurs de substitution de base. Nous avons analysé les erreurs de substitution de base afin de voir si les bases des formes candidates au statut de FdB sont utilisées pour remplacer d'autres bases.

Le tableau suivant présente tous les cas où deux bases verbales se substituent (Tab. 12). Pour obtenir le total de 555 erreurs, nous avons déduit 64 formes où seul le choix de flexion était incorrect du nombre total d'erreurs construites avec des bases existantes (cf. Tab. 8)

Ces résultats montrent que, des deux formes que nous avons retenues (3PR, INF), la base du 3PR est la base substitut la plus fréquente. L'infinitif est également utilisé comme base de construction des formes erronées.

\begin{tabular}{|c|c|c|c|}
\hline Base substitut & Nombre & $\mathbf{\%}$ & Exemple \\
\hline $3 P R$ & 281 & $51.65 \%$ & $/ \mathrm{dwa} / \mathrm{pour} / \mathrm{dwav} /$ \\
\hline INF & 70 & $12.68 \%$ & $/ \mathrm{fEr} / \mathrm{pour} / \mathrm{fõ} /$ \\
\hline PP & 25 & $4.59 \%$ & $/ \mathrm{pri} / \mathrm{pour} / \mathrm{prEn} /$ \\
\hline $4 \mathrm{PR}$ & 90 & $15.62 \%$ & $/ \mathrm{d} / \mathrm{ve} / \mathrm{pour} / \mathrm{dy} /$ \\
\hline $6 \mathrm{PR}$ & 65 & $11.39 \%$ & $/ \mathrm{bwavõ} / \mathrm{pour} / \mathrm{byvõ} /$ \\
\hline $5 \mathrm{PR}$ & 17 & $3.13 \%$ & $/ \mathrm{fEt} / \mathrm{pour} / \mathrm{fõ} /$ \\
\hline PS & 2 & $0.36 \%$ & $/ \mathrm{fi} / \mathrm{pour} / \mathrm{fE} /$ \\
\hline 3 SUBJ & 5 & $0.92 \%$ & $/ \mathrm{fas} / \mathrm{pour} / \mathrm{fõ} /$ \\
\hline Total & $\mathbf{5 5 5}$ & $\mathbf{1 0 0} \%$ & \\
\hline
\end{tabular}

Tab. 12. Classement des erreurs de remplacement de base en fonction de la base substitut

Il est assez facile de voir que les apprenants russes utilisent le 3PR pour construire le paradigme verbal. Cependant, le cas de l'utilisation de l'INF est beaucoup plus ambigu à cause d'un faible taux d'occurrences dans les erreurs de substitution de bases. Pour observer comment l'INF a été utilisé pour construire le paradigme verbal, nous avons analysé plus en détail les erreurs de substitution de bases. Parmi tous les cas d'utilisation de l'INF comme substitut, les trois cibles les plus fréquentes sont le 3FUT (19/70 de remplacements par la base de l'INF : il /dəvwara/ pour il /dəvra/), le 6PR (18/70 : ils /prãd/ pour ils /prEn/) et le 4PR (16/70 : nous /prãdõ/ pour nous /prənõ/).

Dans l'analyse qui suit nous nous sommes concentrés sur les substitutions de bases où deux conditions ont été remplies : 1 / une base existante a été utilisée ; 2 / une flexion appropriée a été utilisée.

Lorsque la cible est le 6PR, les deux conditions sont réunies dans 110/200 erreurs (55\%), alors que l'infinitif n'a été utilisé que dans 11 erreurs (10\%). Globalement, la flexion du 4PR est correctement choisie : 112/152 soit 73.6\% d'erreurs sont marquées avec la flexion correcte /õ/. Cependant, l'utilisation 
des bases existantes pour cette cible est rares. En effet, des bases existantes n'ont été utilisées que dans 31 erreurs, la base de l'INF n'a été utilisée que dans 4 erreurs.

Lorsque la cible est le 3FUT, le taux de flexions correctes dans les erreurs construites avec une base existante est de 64\% (31/48). Cela représente $17 \%$ de toutes les erreurs dans le 3FUT (31/179). Nous avons examiné les bases qui ont été utilisées pour substituer les bases du futur simple. Comme nous pouvons voir dans le tableau suivant, la base de l'INF est le substitut le plus fréquent pour remplacer la base du futur simple (Tab. 14). Les erreurs dans le 3FUT représentent donc le cas le plus saillant d'utilisation de la base de l'INF pour construire le paradigme verbal.

\begin{tabular}{|c|c|c|}
\hline Base substitut & Nombre & Exemple \\
\hline INF & 19 & /fEra/ pour /fəra/ \\
\hline 4PR/IMP & 8 & /byvra/ pour /bwara/ \\
\hline 6PR & 2 & /bwavra/ pour /bwara/ \\
\hline 3PR & 2 & $/ \mathrm{dwara} /$ pour /dəvra/ \\
\hline Total & $\mathbf{3 1}$ & \\
\hline
\end{tabular}

Tab. 14. Erreurs de remplacement de base dans les formes du futur simple si la flexion du futur est maintenue

Pour étayer l'hypothèse que le futur simple est construit à partir de l'INF, il semble intéressant de retourner à l'analyse des productions justes et la détailler pour une seule cible - le 3FUT. Nous avons réanalysé les taux de sélection de bases justes du 3FUT dans deux groupes de verbes : d'un côté, les verbes qui utilisent la même base pour les formes de l'INF et du futur, et de l'autre côté, les verbes où ces deux formes se construisent avec deux bases différentes. Dans notre tâche, cette distinction oppose les verbes prendre, craindre, boire et traduire qui utilisent une seule base pour le futur et l'infinitif aux verbes faire et devoir qui utilisent deux bases différentes. Les taux moyens de sélection de bases correctes dans ces deux groupes de verbes sont résumés dans le Tableau 15.

\begin{tabular}{|c|c|c|}
\hline Niveau & $\begin{array}{c}\text { Taux de sélection de } \\
\text { bases correctes : } \\
\text { verbes à une base }\end{array}$ & $\begin{array}{c}\text { Taux de sélection de } \\
\text { bases correctes : } \\
\text { verbes à deux bases }\end{array}$ \\
\hline N1 & $61.38(14.95)$ & $38.25(25.65)$ \\
\hline N2 & $76.75(13.36)$ & $86.75(5.32)$ \\
\hline
\end{tabular}

Tab. 15. Taux de sélection de bases justes du futur simple dans les verbes qui utilisent une même ou deux bases pour les formes du futur simple et l'infinitif (moyennes et écarts-types).

Nous avons comparé les taux de sélection de bases correctes dans ces deux groupes de verbes. Dans les production du groupe $\mathrm{N} 1$, nous avons observé une tendance à un plus haut taux de correction dans les verbes qui utilisent une même base pour le futur simple et l'infinitif ( $(=2.006, p=0.072)$. Cette tendance n'est, néanmoins, pas présente dans les productions du groupe $\mathrm{N} 2(\mathrm{t}=1.414, \mathrm{p}=0.188)$.

Pour vérifier si le plus haut taux de sélection de bases correctes dans les verbes qui utilisent une seule base pour l'infinitif et le futur simple dans les production du groupe N1 peut être expliqué par leur fréquence plus élevée, nous avons comparé les fréquences moyennes des bases des deux groupes de verbes dans le corpus NSF correspondant à l'input du groupe N1. La fréquence moyenne des bases du futur simple dans les verbes qui utilisent deux bases différentes est de $39.38(\mathrm{SD}=25.50)$ sur 100.000 mots. Ce taux n'est pas significativement différent de la fréquence moyenne des bases du futur simple des verbes qui utilisent une même base pour le futur simple et l'infinitif $(\mathrm{M}=27.21, \mathrm{SD}=21.11)(\mathrm{t}=0.882$, $\mathrm{p}=0.398$ ). Ce résultat suppose que la fréquence ne peut pas expliquer un plus haut taux de sélection de bases correctes du futur simple si elles sont identiques aux bases de l'infinitif dans les productions du groupe N1. 
De manière générale, l'application des critères proposés par Bybee (1985) pour définir les $\mathrm{FdB}$ du paradigme verbal nous amène à la conclusion que les bases du 3PR et de l'INF jouent ce rôle chez les apprenants russes du FLE.

\section{Conclusion}

Dans cet article, nous avons analysé la production des formes verbales du français L2 par des étudiants russophones avec pour objectif d'observer l'influence de la fréquence des formes et des bases. L'analyse primaire des formes justes et des erreurs a montré que la sélection de base est plus lente à acquérir que le marquage par une flexion prononcée. Nous avons observé comment la fréquence des tokens et la présence des formes de base du paradigme verbal influence la sélection de bases.

Premièrement, nous avons tenté de déterminer le rôle de la fréquence des tokens dans la production des formes fléchies et la sélection des bases verbales.

- Nous nous sommes demandés si la fréquence des formes et des bases influençait leur production correcte. Effectivement, les formes et les bases des verbes à fréquence cumulée élevée sont significativement mieux produites que celles des verbes à basse fréquence cumulée. Plus précisément, l'analyse des corrélations entre les fréquences et les taux de production juste a montré que les formes du 4PR, du 6PR et du 3SUBJ sont mieux produites si elles sont plus fréquentes.

- Nous voulions savoir si le niveau des apprenants en français avait une influence sur l'ampleur de l'effet de fréquence. La comparaison des taux de production juste des verbes à haute et à basse fréquence cumulée a montré que les verbes fréquents sont significativement mieux réussis que les verbes rares par les apprenants les plus avancés (niveau N2). Cette différence n'est pas présente dans les productions du niveau le moins avancé $(\mathrm{N} 1)$. L'analyse des corrélations fréquence - taux de productions justes a montré une évolution significative entre les niveaux des apprenants. La corrélation fréquences des formes et des bases - taux de formes justes est plus forte au N2 qu'au N1.

- Nous avons également analysé les formes erronées pour voir si l'effet de la fréquence des formes et des bases s'appliquait également aux remplacements par des formes existantes et aux remplacements des bases verbales. Les formes et les bases substituts à structure simple (patterns FS pour FS et FS pour FC) sont significativement plus fréquentes que les formes et les bases substituées. Lorsque le substitut est une forme complexe (patterns FC pour FC et FC pour FS), sa fréquence de surface n'était pas plus élevée que celle de la cible. Cependant, notons que dans le cas du pattern FC pour FC, la base substitut était plus fréquente que la base cible.

Pour résumer, nous pouvons voir que la sélection des bases verbales est sujette à l'effet de fréquence même dans le cas des formes où la sélection d'une variante de base est cruciale pour exprimer le temps, le mode et la personne (p.ex. le subjonctif présent, le participe passé).

Deuxièmement, en nous appuyant sur les critères de Bybee (1985), nous avons essayé de voir si le verbe français possède réellement une forme de base pour les apprenants russe du FLE.

- Pour identifier quelles formes peuvent avoir le statut de forme de base lors de l'acquisition du FLE, nous avons analysé les formes cibles de notre tâche à la lumière de l'idée que la forme de base doit être peu marquée sémantiquement, fréquente, acquise très tôt et utilisée pour construire le paradigme verbal (Bybee, 1985). Tout d'abord, l'analyse des productions justes a montré que trois formes se distinguent par un taux de juste élevé : le 3PR, l'INF et le PP, et ce pour les apprenants des deux niveaux. Cependant, malgré sont taux de correction élevé, le PP est sémantiquement marqué. Ensuite, l'analyse des erreurs de substitutions de bases a montré que le 3PR est souvent utilisé pour remplacer directement d'autres formes. L'INF est peu utilisé pour remplacer les formes en tant que telles mais s'emploie comme base pour construire des formes du futur à flexion prononcée.

Ces résultats rejoignent le modèle linguistique de Bonami \& Boyé (2003) ainsi que le modèle développemental de Bybee puisque les deux prédisent le statut basique du singulier présent. 
Pour ce qui est des essais d'appliquer la notion de forme de base à l'acquisition du FLE, Bartning (1998) propose également le statut de forme de base du 3PR en précisant que cette forme est utilisée par des apprenants suédois pour remplacer le 6PR ou le 1PR. Par ailleurs, Bartning signale la présence d'une seconde forme de base - une forme de base longue qui correspond à la forme du 6PR des verbes irréguliers (p.ex. il /dorm/). Hebdor (2003) explique ce type d'erreurs comme conjugaison par analogie et non pas comme une manifestation du statut basique du 6PR, d'autant plus que les formes longues utilisées pour remplacer le singulier présent ne relèvent pas toujours du 6PR : il /prãd/. Rappelons que le 6PR ne remplit pas le deuxième critère de forme de base proposé par Bybee (1985) : en effet, cette forme n'est pas acquise tôt, surtout dans les verbes irréguliers. Cette idée a été bien illustrée sur l'exemple des étudiants suédois : la production systématique des 6PR irréguliers correctes n'arrive qu'au niveau avancé des apprenants (Bartning, 2004). Les résultats de notre tâche rejoignent ces données. Rappelons que les verbes élicités dans la présente étude sont les verbes où la base du 6PR est justement une base longue (à part faire). Le 6PR est une des formes les moins réussies, sa production correcte est en forte corrélation avec sa fréquence de surface, ce qui va à l'encontre des critères de forme de base.

Les résultats similaires aux nôtres quant au statut des formes de base du 3PR et de l'INF ont été obtenus par Thomas (2005) qui a étudié l'acquisition de la morphologie verbale française par des apprenants suédois. Il est donc possible de supposer que le statut basique du 3PR et de l'INF peut être expliqué par la structure de la langue française vu que des résultats pareils ont été obtenus pour des apprenants avec des L1 différentes.

Pour conclure, ces résultats sont d'autant plus intéressants si nous gardons à l'esprit qu'en français, le choix d'une variante de base verbale peut être crucial pour exprimer les différences de personne, du temps ou du mode dans le cas de la flexion zéro. Nous avons montré que la sélection des bases verbales est influencée par au moins deux facteurs différents des notions du temps verbal, de la personne ou du mode, à savoir la fréquence des tokens et le statut de formes de base du 3PR et de l'INF. Autrement dit, même si les apprenants du FLE ont déjà acquis les différences conceptuelles entre les catégories verbales, la production des formes de surface correspondantes peut être facilitée ou inhibée par la fréquence des cibles ainsi que des autres bases compétitives du même verbe et, d'autre part, par le fait que la base cible est différente ou identique à une des formes de base.

\section{Références bibliographiques}

Ågren M. (2005). La morphologie du nombre dans le syntagme verbal en français L2 écrit. L'accord de la $3^{\text {ème }}$ personne du pluriel. Acquisition et production de la morphologie flexionnelle. Actes du Festival de la morphologie. PERLES : petites études romanes de Lund. Extra seriem 20. 131-150.

Bartning I., Schlyter S. (2004). Itinéraires acquisitionnels et stades de développement en français L2. Journal of French Language Studies. 14 (3). 281-299.

Bartning I. (1998). Procédés de grammaticalisation dans l'acquisition des prédictions verbales en français parlé. Travaux de linguistique. 36. 223-234.

Bonami O., Boyé G. (2003). Supplétion et classes flexionnelles dans la conjugaison du français. Langages 152. 102126.

Bonami O., Boyé G. (2003). Suppletion and dependency in inflectional morphology. In Frank van Eynde, Lars Hellan, et Dorothee Beermann (éds.), Proceedings of the HPSG-2002 Conference. Stanford: CSLI Publications.

Boyé G. (2000). Problèmes de morpho-phonologie verbale en français, en espagnol et en italien. Thèse présentée en vue de l'obtention du grade de docteur de l'Université Paris VII - Denis Diderot.

Bybee J. L. (1985). Morphology. A study of the relation between meaning and form. Amsterdam: John Benjamins.

Bybee J. L. (1991). Natural morphology : the organization of paradigms and language acquisition. In: Huebner \& Ferguson (éd.): Crosscurrents in second language acquisition and linguistic theories. Amsterdam: John Benjamins. 67-91. 
Bybee J. L., Hopper P. (éd.) (2001). Frequency and the emergence of linguistic structure. Amsterdam : John Benjamins.

Debrenne M. (2006). La langue française dans la pratique langagière des Russes. Déviatologie interlinguistique. (Franzuzskij yazyk $v$ retchevoj praktike russkikh : mejyazykovaya deviatologiya). Novossibirsk : Novossibirskij gosudarstvennyj universitet.

Ellis N. C. (2002). Frequency effets in language processing : A review with implications for theories of implicit and explicit language acquisition. Studies in second language acquisition. 24 (2). 143-188.

Gor K., Chernigovskaya T. (2003). Formal instruction and the mental lexicon: The acquisition of verbal morphology. Instructed second language learning. Brussel : Vrie Universiteirt. 103-136.

Granget C. (2005). Développement de l'accord verbal avec un sujet pluriel dans les récits écrits d'apprenants germanophones scolarisés en français. Acquisition et production de la morphologie flexionnelle. Actes du Festival de la morphologie. PERLES : petites études romanes de Lund. Extra seriem 20. 111-124.

Hedbor C. (2005). Le traitement implicite et explicite de l'inflexion de la $3^{\text {ème }}$ personne du pluriel du verbe chez les apprenants guidés du français LE. Acquisition et production de la morphologie flexionnelle. Actes du Festival de la morphologie. PERLES : petites études romanes de Lund. Extra seriem 20. 151-166.

Hedbor C. (2003). Les règles d'abord ou la production directe du français écrit? Etude longitudinale sur l'utilisation ou non des connaissances explicites par cinq élèves de différentes attitudes cognitives (licentiatavhandling). : petites études romanes de Lund. Extra seriem 18.

Langacker R. (1987). Foundations of Cognitive Grammar, Vol. 1. Theoretical Prerequisites. Stanford: Stanford University Press.

Marcus G.F., Pinker S., Ullman M., Hollander M., Rosen T.J., Xu F. (1992). Overregularization in language acquisition. Monographs of the Society for Research in Child Development. 57(4). Serial No. 228.

Meunier F., Marslen-Wilson W. (2000) Regularity and Irregularity in French Inflectional Morphology. Proceeding of the $22^{\text {nd }}$ Annual Meeting of the Cognitive Science Society. N.J.: LEA. 346-351.

Matcovich O. (1998). Regular inflection in the mental lexicon: Evidence from Italian. Proceedings from the conference "The Verb in Cognitive Grammar".

New B., Pallier C., Ferrand L. et R. Matos. (2001). Une base de données lexicales du français contemporain sur internet: LEXIQUE. Année Psycholinguistique. 101. 447-462. http://www.lexique.org

Payre-Ficout C. (2007). L'apprentissage du prétérit et du present perfect dans le cadre scolaire: etude extensive chez des apprenants francophones du secondaire et des étudiants du supérieur. Thèse présentée en vue de l'obtention du grade de docteur de l'Université Stendhal Grenoble III.

Pinker S. \& Prince A.S. (1988). On language and connectionism: Analysis of a parallel distribued processing model of language acquisition. Cognition. 28. 73-193.

Pinker S. (1991). Rules of language. Science. 253. 530-535.

Pinker S. \& Prince A.S. (1991). Regular and irregular morphology and the psychological status of rules of grammar. Proceedings of the 17th Annual Meeting of the Berkley Linguistic Society. Berkley, CA: Berkley Linguistic Society.

Ragnasdottir H., Simonsen H.G., Plunkett K. (1997). Acquisition of the past tense in Icelandic and Norwegian Children. Proceedings of the $28^{\text {th }}$ Annual Child Language Research Forum. Stanford.

Schlyter S. (2005). Formes verbales au pluriel à l'oral plus marquées chez les apprenants guidés que non-guidés ? Acquisition et production de la morphologie flexionnelle. Actes du Festival de la morphologie. PERLES : petites études romanes de Lund. Extra seriem 20. 125-130.

Simonsen H.G. Past tense acquisition and processing in Norwegian: Experimental evidence. Language and Language Behavior. 3 (2). 86-101.

Thomas A. (2005). Formes ambiguës en -E: input et categories sémantiques. Acquisition et production de la morphologie flexionnelle. Actes du Festival de la morphologie. PERLES : petites études romanes de Lund. Extra seriem 20.35-52. 